\title{
Next-generation laser-based photodynamic endoscopic diagnosis using 5-aminolevulinic acid for early gastric adenocarcinoma and gastric adenoma
}

\author{
Takuki Sakaguchi, Hidehito Kinoshita, Yuichiro Ikebuchi, Tsutomu Kanda, Taro Yamashita, \\ Hiroki Kurumi, Masashi Fujii, Mirai Edano, Takashi Hasegawa, Takumi Onoyama, Akira Yoshida, \\ Koichiro Kawaguchi, Kazuo Yashima, Hajime Isomoto
}

Tottori University Faculty of Medicine, Japan

\section{Abstract}

\begin{abstract}
Background Photodynamic diagnosis (PDD) is an optical imaging technology based on the fundamental biological features of porphyrin metabolized in cancer cells. We reported the usefulness of laser-based photodynamic endoscopic diagnosis (LPDED) with 5-aminolevulinic acid (5-ALA) for early gastric cancers. However, the first-generation prototype endoscope system had the flaw that the images captured were rather dark. To overcome this, we constructed a nextgeneration endoscope system for LPDED.

Methods We evaluated the usefulness of the next-generation prototype endoscope system, called Sie-P2, for brighter LPDED to detect early gastric cancer (EGC) and gastric adenoma. The 14 patients diagnosed with EGC and/or gastric adenoma who underwent endoscopic submucosal dissection (ESD) at our hospital between April 2018 and March 2019 were enrolled consecutively in this study. Patients were administered 5-ALA orally and LPDED was performed $3 \mathrm{~h}$ later. The primary endpoint was the presence of fluorescence in tumors when we performed LPDED. The secondary endpoint was to assess the adverse events related to each LPDED procedure.
\end{abstract}

Results One patient was excluded because of a contraindication, while the remaining 13 patients (median 72 years, range 56-77; one female) with 16 lesions were assessed. There were 10 elevated lesions and $6 \mathrm{flat} /$ depressed lesions; there were 10 EGCs and 6 adenomas. LPDED-fluorescence was detected in all 16 lesions (sensitivity 100\%, 95\% confidence interval 79-100\%). Two cases showed temporary, though not substantial, elevation in blood liver function tests.

Conclusion All lesions examined were LPDED-positive, indicating that the Sie-P2 system could be useful.

Keywords Photodynamic diagnosis, 5-aminolevulinic acid, gastric cancer, gastric adenoma

Ann Gastroenterol 2020; 33 (3): 1-8

\section{Introduction}

Gastric cancer has been to shown to be the fifth most common cancer and the third leading cause of cancer-related

Division of Medicine and Clinical Science, Tottori University Faculty of Medicine, Tottori, Japan

Conflict of Interest: None

Correspondence to: Hajime Isomoto, $\mathrm{MD}, \mathrm{PhD}$, Division of Medicine and Clinical Science, Tottori University Faculty of Medicine, 36-1, Nishi-cho, Yonago, Tottori, 683-8504 Japan,

e-mail: hajimei2002@yahoo.co.jp

Received 28 September 2019; accepted 11 March 2020; published online 13 April 2020

DOI: https://doi.org/10.20524/aog.2020.0479 death worldwide [1]. When early gastric cancer (EGC) can be identified, endoscopic submucosal dissection (ESD) may be performed with adequate survival rates [2-4]. On the other hand, there is still a high mortality rate for advanced gastric cancer. Therefore, it is of clinical importance to detect it in its early stage.

In recent years, remarkable advances in endoscopic imaging technologies, such as narrow band imaging (NBI) and blue laser imaging (BLI), have made it easy to identify EGC. However, the detection rate of EGC is still insufficiently low, as even experienced endoscopists cannot always identify EGC against an inflammatory and atrophic background mucosa, or following Helicobacter pylori eradication spreading through the gastric cavity. Yamaguchi et al reported that the detection rate of white light imaging (WLI) is $70.3 \%$. Even if WLI is combined with NBI or BLI, the detection rates are $80.0 \%$ and $86.7 \%$, 
respectively [5]. Menson et al reported that $11.4 \%$ of upper gastrointestinal cancers had been missed during esophagogastroduodenoscopy (EGD) within the past 3 years [6]. Shimodate et al reported that $75.2 \%$ of EGC cases had not been recorded in the previous EGD report, but were evident upon review of endoscopic photographs [7].

To overcome such issues, we reported the utility of laserbased photodynamic endoscopic diagnosis (LPDED) with 5-aminolevulinic acid (5-ALA), using a prototype endoscope system (Sie-P1, Fujifilm Co., Tokyo, Japan) [8]. 5-ALA is a natural amino acid that has been used in the photodynamic diagnosis (PDD) of several tumors, such as brain glioma [9] and bladder cancer $[10,11]$. The 5-ALA is metabolized into protoporphyrin IX (PpIX) in the heme biosynthesis pathway, and PpIX is synthesized in mitochondria and tends to accumulate in cancer cells, emitting red fluorescence (635 $\mathrm{nm}$ light) when irradiated by blue wavelength light (375-440 nm) [12,13]. LPDED, a 5-ALA-based PDD, is an optical imaging technology based on the fundamental biological features of porphyrin metabolism in cancer cells [14]. The red fluorescence helps us detect certain tumor tissues in real time during operation or ongoing endoscopy. In recent years, 5-ALA became on-label as a sole photosensitizer for PDD in the field of glioblastomas and bladder cancers and is covered by Japanese health insurance. It has been investigated in a doctor-initiated clinical trial for the detection of peritoneal dissemination of gastric cancers during preoperative surgical laparoscopy.

In the Sie-P1 prototype, intact laser light with a wavelength of $410 \mathrm{~nm}$, readily available commercially, was employed for excitation. The power, however, was insufficient to detect the fluorescence of PpIX intensely. Moreover, the endoscope, XG-0002-P1, had a built-in cut filter to exclude excitation light during observation of the PpIX fluorescence. There was an additional drawback in that the images captured were rather dark, because the presence of the cut filter meant that only the PpIX fluorescence could be received (Fig. 1) [8].

Therefore, we developed a novel prototype endoscope system (Sie-P2) to overcome these drawbacks and to improve the dark imaging so that no cut filter would be required. Our purpose, in this pilot study, was to examine the sensitivity and safety of LPDED with the Sie-P2 system in patients with differentiated EGCs or adenoma.
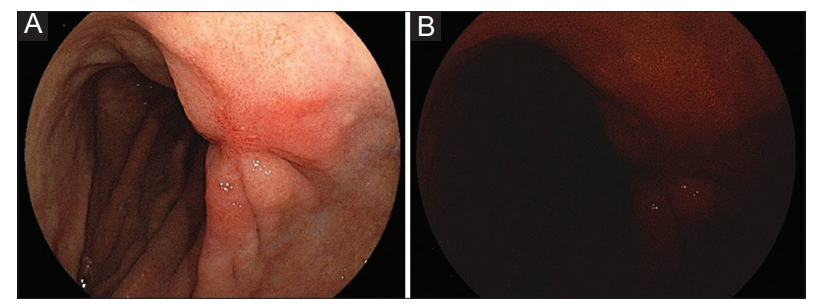

Figure 1 (A) A IIc-type lesion was located on the posterior wall of the middle third of the stomach (white light observation). (B) A red fluorescent but dark picture of the lesion was captured using laserbased photodynamic endoscopic diagnosis with the first prototype, Sie-P1

\section{Patients and methods}

\section{Study population}

In this study, 14 consecutive patients undergoing ESD for gastric epithelial neoplasms were enrolled at our hospital between April 2018 and March 2019. Patients who satisfied the following criteria were included: 1) patients aged 20 years or older who gave their consent to this study; 2) patients candidates for an endoscopy examination; and 3) patients whose Eastern Cooperative Oncology Group Performance Status (PS) was 0 or 1 . The exclusion criteria were as follows: 1) allergy to 5-ALA; 2) porphyria; 3) taking medicine known to cause photosensitivity (e.g., tetracycline, sulfonamide, new quinolone, piperacillin) or foods containing St. John's wort; 4) anticancer drug treatment within 1 month before signing the consent form; 5) presence of another tumor; 6) taking anticoagulant medication; 7) pregnancy or possibility of pregnancy; 8) acute inflammation; 9) severe heart, liver or renal dysfunction; 10) very poor general condition, respiratory disease, stenosis or large ulcers, so that endoscopy would be considered dangerous; and 11) attending physician's judgment.

The study was approved by the institutional review board (\#17B013), and written informed consent was obtained from all included patients prior to their enrolment in the study.

\section{5-ALA LPDED}

Three hours after the oral administration of 5-ALA in a dose of $20 \mathrm{mg} / \mathrm{kg}$, we inserted the second-generation prototype endoscope, Sie-P2, in WLI mode. Once lesions were detected, WLI mode was switched to PDD mode. When the fluorescence signal was visualized and was confined to the tumor, but not the surrounding non-tumorous tissue, it was classed as LPDED-positive.

After the observation of the lesion, we observed the cavity of the stomach in PDD or WLI mode. After observation of the stomach cavity, we exchanged scope (GIFQ260J, Olympus Co., Tokyo) to perform ESD on the target lesions. Thus, LPDED was conducted on the day of ESD and just before its performance in each case.

\section{Endoscopic system (Fig. 2)}

We developed a novel second-generation prototype endoscope system for LPDED, called the Sie-P2 system (FUJIFILM Co., Tokyo, Processor: VP-7000-P2, Light source: LL-7000-P2, EGD scope: EG-L590ZW). LL-7000-P2 was constructed by adjusting a LASEREO7000 to irradiate with laser light at $410 \mathrm{~nm}$, more strongly (around 2-fold) than the LASEREO4450 that formed the basis of the Sie-P1 system. We believed that strong irradiation with this $410 \mathrm{~nm}$ laser would achieve powerful excitation light and thus strong PpIX fluorescence emission. The EG-L590ZW is a commercially available endoscope that does not have a cut filter to exclude 


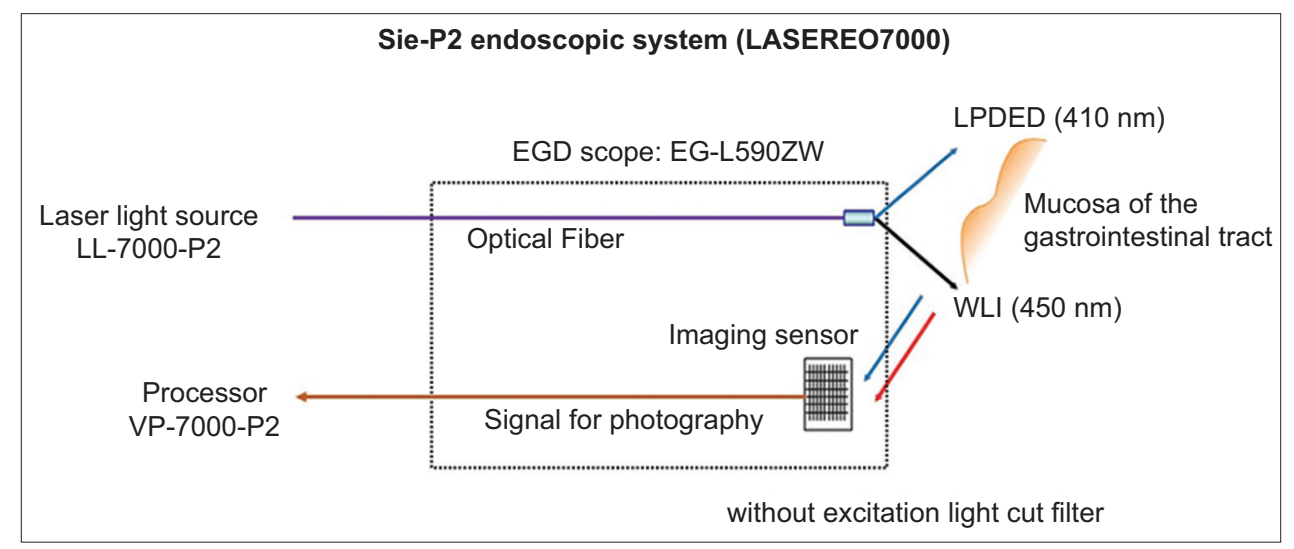

Figure 2 The second-generation prototype endoscope system for laser-based photodynamic endoscopic diagnosis (LPDED), Sie-P2 system, made by adjusting a LASEREO7000. The system consists of light source, LL-7000-P2, processor, VP-7000-P2, and esophagogastroduodenoscopy (EGD) scope, EG-L590ZW. EG-L590ZW is a commercially available endoscope including white light imaging (WLI), which does not have a cut filter to exclude excitation light

excitation light. Adjustment of the white balance was required to achieve the preferred color inside the processor. With this prototype endoscope system, by pushing a button it is easy to switch between the WLI, BLI, and PDD modes in turn.

\section{Histopathological evaluation}

The lesions resected during ESD were classified using the $15^{\text {th }}$ Edition of the Japanese Classification of Gastric Carcinoma [15]. We recorded the tumor characteristics, including tumor location, macroscopic type of tumor, size, histology and the depth of tumor invasion. Tumor location was categorized into the upper, middle and lower thirds of the stomach. Macroscopic type of tumor was categorized into the elevated type or flat/depressed type. The histological classification included adenoma, differentiated adenocarcinoma (well [tub1] or moderately differentiated adenocarcinoma [tub2] or papillary adenocarcinoma [pap]), or undifferentiated adenocarcinoma (poorly differentiated adenocarcinoma [por] or signet-ring-cell carcinoma [sig]). Again, when we found new PDD-positive lesions, we performed biopsy sampling and subjected the samples to histopathological evaluation.

\section{Evaluation items}

The primary endpoint was the presence of fluorescence in tumors when we performed LPDED. Patients were administered 5-ALA and LPDED was performed $3 \mathrm{~h}$ later. After observation of the lesion with LPDED, we performed ESD and the specimens were evaluated histopathologically. The secondary endpoint was to assess the adverse events related to oral intake of 5-ALA and the LPDED procedure. We checked each patient's vital signs and symptoms, and laboratory tests were performed before and after LPDED in each patient. More specifically, we assessed adverse events, including fever, hypothermia, hypotension, non-cardiac chest pain, abdominal pain, nausea, vomiting, photosensitivity, anemia, leukocytosis, thrombocytopenia, as well as abnormalities in blood chemistry, including aspartate aminotransferase, alanine aminotransferase, alkaline phosphatase, total bilirubin creatinine, potassium, and sodium. The grade of each adverse event was classified using Common Terminology Criteria for Adverse Events (CTCAE) grades

(http://www.jcog.jp/doctor/tool/CTCAEv5J_20180730_ v21_0.pdf).

\section{Statistical analysis}

The statistical significance of comparisons between the groups was determined using the $\chi^{2}$ test, and the Student's $t$-test. P-values $<0.05$ were considered significant.

\section{Results}

\section{Clinicopathological characteristics}

After enrolment, 1 patient who had been taking anticancer drug treatment within 1 month before signing the consent form was excluded, in accordance with the exclusion criteria. The remaining total of 13 patients, with 16 lesions, were included in this study. Table 1 shows the characteristics of the patients and lesions. The patients' median age was 72 years (range 5677 ), there were $12 \mathrm{men}$, and all of the patients had a PS grade of 0. A total of 16 lesions, 10 adenocarcinomas and 6 adenomas, were included in the analysis. There were 10 elevated lesions ( 9 lesions were 0-IIa; one lesion was 0-I macroscopic type) and 6 flat/depressed lesions (all lesions were 0-IIc macroscopic type). Ten lesions were adenocarcinomas, of which 9 were intramucosal adenocarcinoma and 1 had infiltrated into the submucosa with lymphovascular involvements, and 6 lesions were adenomas. Of the 10 adenocarcinomas, 6 lesions were 
Table 1 Characteristics of patients $(n=13)$ and lesions $(n=16)$

\begin{tabular}{lc} 
Characteristics of lesions & $(\mathrm{n}=16)$ \\
\hline Tumor location & $1 / 12 / 3$ \\
$\quad$ Upper/middle/lower & \\
Macroscopic type of tumor & $10 / 6$ \\
$\quad$ Elevated/flat or depressed & \\
Histology & 10 \\
$\quad$ Differentiated & 0 \\
Undifferentiated & 6 \\
Adenoma & \\
Depth of tumor invasion & $15 / 1$ \\
Intramucosal/submucosal & \\
New lesions & $1 / 1 / 1$ \\
$\quad$ Adenoma/tubular adenocarconima/non-neoplastic \\
\hline
\end{tabular}

well-differentiated (tub1), 3 were differentiated (tub1+tub2), and 1 lesion was mixed type (tub1+tub2>por). The average tumor size of the excised lesions was $18.9 \mathrm{~mm}$ in diameter. In this case series, ESD was performed successfully and uneventfully in all patients. Since 6 lesions were diagnosed as adenomas, we performed ESD for the purpose of total biopsy, considering the known risks of malignant transformation and the possibility of malignant foci in the precancerous lesion [16].

\section{Detection rate of LPDED with the second-generation endoscope}

In this study, ALA-mediated PpIX fluorescence was detected in all 16 lesions by LPDED, giving a sensitivity of $100 \%$ (95\% confidence interval [CI] 79-100\%). Fig. 3 shows a representative case. The patient was 72 years old. The slightly reddish and elevated lesion was located on the anterior wall of the lower gastric body (Fig. 3A) and the maximum tumor diameter was $15 \mathrm{~mm}$. We were able to detect orange fluorescence with LPDED (Fig. 3B) and removed the lesion using the ESD method on the same day. The lesion was revealed to be an intramucosal well-differentiated adenocarcinoma.

\section{Intensity levels of fluorescence of 5-ALA-mediated PDD, LPDED with the Sie-P2 system}

Since there was a difference in the level of fluorescence among the 16 lesions, we divided the LPDED-positive lesions into LPDED-(++) and LPDED-(+); the tumor was deemed LPDED- $(++)$ when the intense and diffuse fluorescence was depicted; the tumor was deemed LPDED-(+) when weak and/or focal fluorescence was depicted [8]. Fig. 4 shows the representative LPDED- $(++)$ showing intense and diffuse red fluorescence compared to LPDED-(+) showing weak red fluorescence. Two doctors (TS and HI) engaged in endoscopic practice decided whether the lesions were LPDED- $(++)$ or LPDED- $(+)$ ( $\kappa$ value $=1.00, \mathrm{P}=0.00011,95 \%$ CI $0.4939-1.00)$. There were 9 LPDED-(++) and 7 LPDED-(+) lesions detected

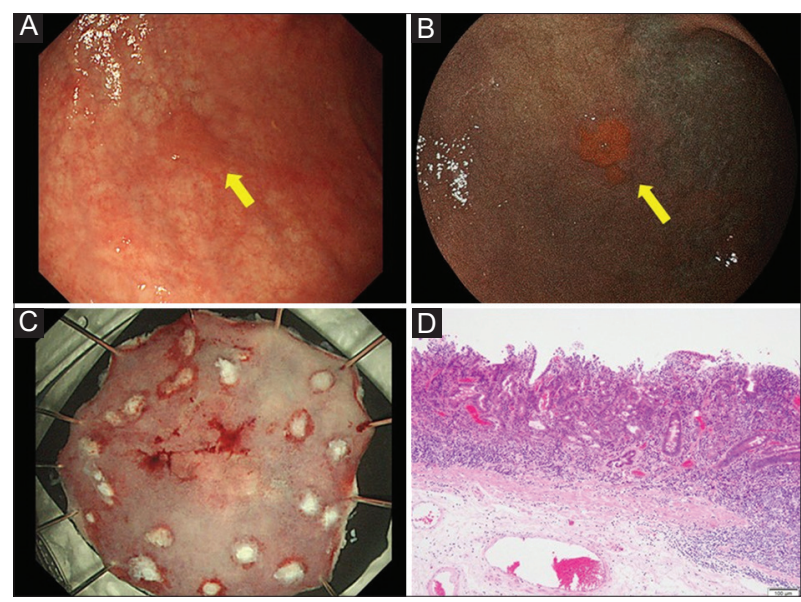

Figure 3 The reddish and slightly elevated lesion with equivocal demarcation was located on the anterior wall of the lower gastric body and the maximum tumor diameter was $15 \mathrm{~mm}$ (A). We could detect fluorescence using laser-based photodynamic endoscopic diagnosis with Sie-P2 (B). (C) We conducted endoscopic submucosal dissection. The resected specimen revealed intramucosal differentiated adenocarcinoma (D)

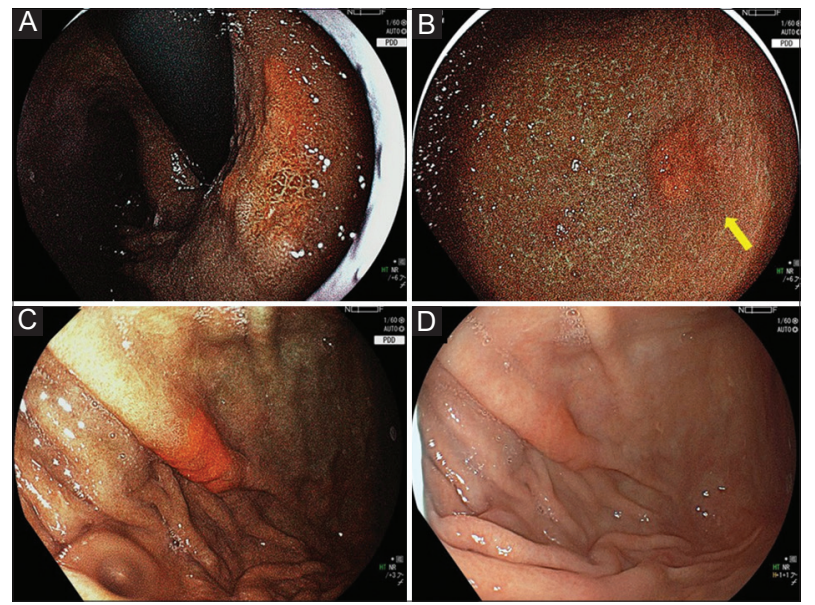

Figure 4 (A) The tumor was deemed laser-based photodynamic endoscopic diagnosis (LPDED)-(++); the intense and diffuse fluorescence was depicted was depicted; LPDED-positive $(+)$ when the weak or focal fluorescence was depicted (B). The LPDED-(++) lesion was located on the lesser curvature of the middle third of the stomach, where there was intense and diffuse fluorescence (A), while the LPDED-(+) lesion was located on the anterior wall of the gastric lower body (B) in the same patient. (C) The representative LPDED-(++) lesion was clearly visible compared to white light imaging (D)

(Table 2 [8]). As for macroscopic type, the 10 elevatedtype tumors included $4(+)$ and $6(++)$, while the 6 flat or depressed type tumors included 3 of each. There were $4(+)$ and $6(++)$ among the differentiated adenocarcinomas, while the adenomas included 3 of each.

On the other hand, among the 16 lesions, there were 5 indistinct lesions with equivocal margins on WLI observation. Among these 5 WLI-indistinct lesions, 4 were classified as LPDED-(++), while 1 was LPDED-(+). However, the current results regarding the diagnostic ability of LPDED were 
Table 2 Intensity levels of fluorescence and macroscopic and microscopic type of lesions

\begin{tabular}{ll}
\hline Characteristics of lesions & $\begin{array}{l}\text { Intensity levels of LPDED } \\
\text { fluorescence }(\mathrm{n})\end{array}$ \\
\hline Macroscopic type of tumor & $\begin{array}{l}\text { Elevated (10) } \\
+: 4 ;++: 6\end{array}$ \\
Elevated/flat or & Flat or depressed (6) \\
depressed & $+: 3 ;++3$ \\
& Differentiated adenocarcinoma (10) \\
& $+: 4 ;++: 6$ \\
Histology & Adenoma $(6)$ \\
Differentiated & $+: 3 ;++: 3$ \\
adenocarcinoma/ &
\end{tabular}

The tumor was deemed laser-based photodynamic endoscopic diagnosis (LPDED)-positive (++) when the intense and diffuse fluorescence was depicted; LPDED-positive $(+)$ when the weak or focal fluorescence was depicted

semi-quantified and seemingly subjective and might be tentative because of the small number of patients in this case series.

There was significant difference $(\mathrm{P}<0.0001)$ in the fluorescent intensities between tumors and non-tumorous surrounding regions: mean 67.8 (range 62.8-73) and mean 19.6 (range 11.4-28.5), respectively. The calculated value (S) had the property in which the value could become maximum for the fluorescence in the area of interest in the tumors and the signal-to-noise ratio (SNR) was the mean calculated $\mathrm{S}$ value in the area of interest in the tumors, divided by the standard deviation of the value (defined as noise, $\mathrm{N}$, which included the fluctuation of the fluorescence intensities in the area of interest). The calculated SNR ranged from 7-17 (mean 11).

\section{New lesions detected with LPDED}

We found 3 new LPDED-positive and possibly neoplastic lesions that could not be definitely identified by conventional WLI during the prior endoscopic examination. Biopsy sampling was performed for each and the samples were subjected to histopathological evaluation. Two of the lesions proved to be neoplastic, while 1 was non-neoplastic; the latter case was deemed a false positive. Fig. 5 shows images of a new lesion. The lesion was located on the greater curvature of the antrum (Fig. 5A). We did not detect the lesion at the initial endoscopic examination. However, during the following endoscopic examination, we were able to detect it, but only using LPDED mode (Fig. 5B). The lesion was removed using ESD on another day (Fig. 5C), and was revealed to be a high-grade adenoma (Fig. 5D). Fig. 6 shows images of a different new lesion. The lesion was located on the anterior wall of the upper third of the body (Fig. 6A). We were able to detect the lesion using LPDED mode for the first time (Fig. 6B), and took a biopsy. The result was tubular adenocarcinoma.

\section{Adverse events}

There was transient elevation of circulating aspartate aminotransferase levels up to over $100 \mathrm{IU} / \mathrm{L}$ (Grade 2, in CATAE

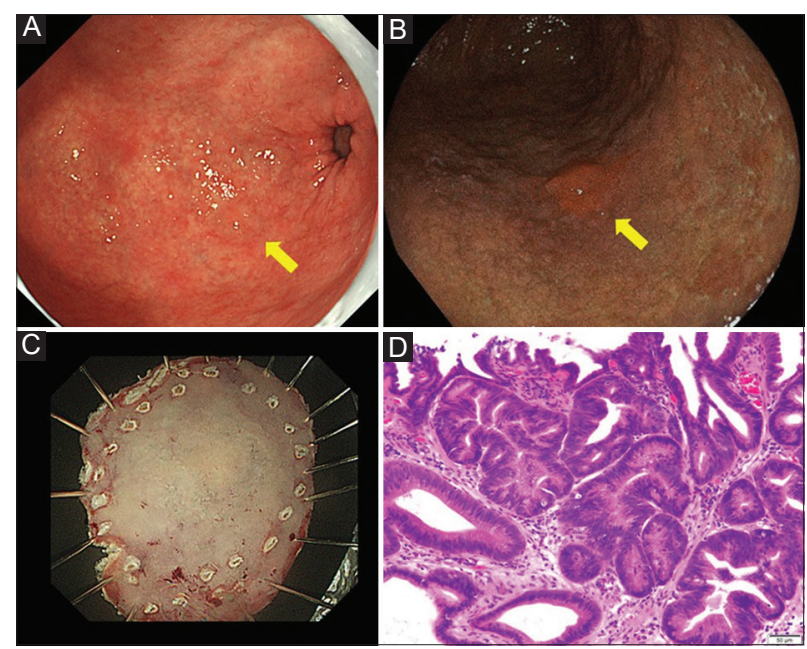

Figure 5 (A) Conventional endoscopic examination carried out 1.5 month before endoscopic submucosal dissection (ESD). We could not detect the 0-IIa lesion on the greater curvature of the pyloric antrum. (B) We could detect the lesion with fluorescence by laser-based photodynamic endoscopic diagnosis (deemed (+)) using the SieP2 system. (C) and (D) We performed ESD later, and revealed a high-grade adenoma

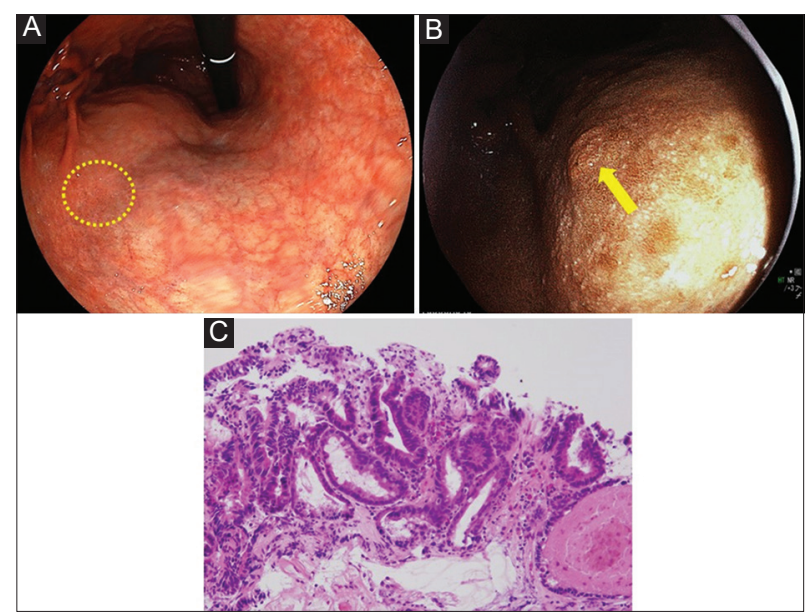

Figure 6 (A) We could not detect this lesion at the first white light imaging observation before endoscopic submucosal dissection. (B) When we performed laser-based photodynamic endoscopic diagnosis (LPDED), we were able to detect the new lesion, located on the anterior wall of the gastric body, despite relatively weak fluorescence, deemed LPDED-(+). The biopsy specimen obtained from this lesion showed tubular adenocarcinoma (C)

v5.0). In addition, 6 cases exhibited transient leukocytosis (Grade 3 in CTCAE v5.0), but during careful follow up the levels returned to normal. Otherwise, there were no substantial changes in symptoms and signs and laboratory data (Table 3).

\section{Discussion}

In the literature concerning the field of diagnosis with a flexible endoscope for gastric neoplasia, there are few 
Table 3 Adverse events evaluated by CTCAE v5.0

\begin{tabular}{|c|c|c|c|c|c|}
\hline \multirow{2}{*}{ Adverse events $(n=13)$} & \multirow{2}{*}{ No complication } & \multicolumn{4}{|c|}{ CTCAEv5.0 } \\
\hline & & Grade 1 & Grade 2 & Grade 3 & Grade 4 \\
\hline Fever & 13 & & & & \\
\hline Hypothermia & 13 & & & & \\
\hline${ }^{\star}$ Hypotension & 11 & 2 & & & \\
\hline Non-cardiac chest pain & 13 & & & & \\
\hline Abdominal pain & 10 & 3 & & & \\
\hline Nausea & 10 & 3 & & & \\
\hline Vomiting & 10 & 3 & & & \\
\hline Photosensitivity & 13 & & & & \\
\hline Anemia & 13 & & & & \\
\hline Leukocytosis & 7 & & & 6 & \\
\hline Thrombocytopenia & 13 & & & & \\
\hline \multicolumn{6}{|l|}{ Blood biochemistry } \\
\hline Aspartate aminotransferase increased & 6 & 5 & 2 & & \\
\hline Alanine aminotransferase increased & 10 & 3 & & & \\
\hline Alkaline phosphatase increased & 13 & & & & \\
\hline Total bilirubin increased & 12 & 1 & & & \\
\hline Creatinine increased & 13 & & & & \\
\hline Hyperkalemia & 12 & 1 & & & \\
\hline Hypokalemia & 13 & & & & \\
\hline Hyponatremia & 13 & & & & \\
\hline
\end{tabular}

publications relating to PDD. Nakamura et al were the first to report the detection of fluorescence in a specimen resected by endoscopic resection, after oral administration of 5-ALA in an EGC [17]. They also reported the usefulness of 5-ALA-PDD using an outside excitation light source in the form of a diode laser probe introduced through a forceps hole [18]. Meanwhile, we had constructed the all-in-one prototype endoscope, Sie-P1, for LPDED, and reported its usefulness for EGCs, in particular differentiated adenocarcinomas [8].

The first-generation endoscope, however, had the inherent flaw that the pictures captured were rather dark, as mentioned above. In the Sie-P1 system, the VP-0001 and LL4450-P1 are equipped and prepared by adjusting a LASEREO 4450 (Processor: VP-4450HD, Light source: LL-4450). The LL-4450-P1 can emit laser light at $410 \mathrm{~nm}$ and $450 \mathrm{~nm}$, and we were able to use the laser light at the shorter wave length for excitation of PpIX, as it was commercially available. On the other hand, the relatively low light power and thereby weakness of the PPIX fluorescence was responsible for the darkness of the fluorescence images seen using Sie-P1 [8].

We therefore constructed a new prototype of a secondgeneration endoscope, called Sie-P2. This system supports the emission of multiple wavelengths of visible light and is equipped with WLI, BLI and PDD modes. Pushing the button of endoscope causes blue light at $410 \mathrm{~nm}$ to be emitted for PDD, with a light power of up to almost twice the intensity possible with Sie-P1. Accordingly, a safety assessment would be required for Sie-P2, and we investigated adverse events in the current case series. These included transient leukocytosis and elevation of circulating aspartate aminotransferase, but during careful follow up monitoring they returned to normal levels. The elevation of white blood cell counts could be a direct effect of the ESD procedure, while the liver dysfunction was attributable to the 5-ALA administration but not to the PDD by itself. The dosage of 5-ALA for oral intake was $20 \mathrm{mg} / \mathrm{kg}$ in water, exactly the same as is approved by health insurance for brain and bladder tumors [10,11]. According to the specifications for 5-ALA, there are a number of possible side effects as follows: nausea (6.7\%), vomiting (4.4\%), fever (4.4\%), liver dysfunction (4.4\%), and thrombocytopenia (2.2\%). These are generally mild and we included them in the assessment of adverse events. One of the most severe adverse event is phototoxicity (0-5.8\%), which can be avoided by light shading. In this study, we implemented light shading during hospitalization for $24 \mathrm{~h}$ after the LPDED procedure, and thus avoided any photosensitivity reaction in all cases. LPDED with Sie-P2 under the study protocol was permissible with regard to safety. It is important to apply light shading for at least 1-2 days. 
In the previous Sie-P1 system, the EGD endoscope, XG-0002-P1 had to be constructed with a sharp cut filter to exclude excitation light from observation of the PpIX fluorescence, and had to be handcrafted in each case, limiting prevalent use. Moreover, this could also be related to the dark image capture, because only the PpIX fluorescence around $635 \mathrm{~nm}$ could be received through the bandpass filter. On the other hand, the cut filter was not required in the second-generation endoscope for LPDED.

In this pilot case series study, we confirmed that the LPDED system could be useful. In this study, all lesions were PDD positive with Sie-P2. Furthermore, we were able to detect 2 new gastric tumorous lesions with LPDED, not detected at the initial endoscopic examination, suggesting that LPDED using 5-ALA could be useful for the screening of gastric neoplasia, even in multiple lesions.

As for the false-positive lesions (images) of PDD by LPDED, when we found 3 new LPDED-positive possibly neoplastic lesions, we performed biopsy sampling in each and subjected them to histopathological evaluation. Two of the lesions proved to neoplastic, while 1 was non-neoplastic, and this case was deemed a false positive. It might be difficult to distinguish intestinal-type gastric tumors from surrounding non-neoplastic background inflammatory and atrophic mucosa, sometimes accompanied by intestinal metaplasia, using LPDED mode. Nevertheless, the current results regarding the diagnostic accuracy of the ALA-mediated PDD method must be considered tentative, because of the small numbers of such cases. To resolve the false-positive issue, further large prospective studies need to be performed with systematic observation of the whole lumen of the stomach, not only focusing on neoplastic lesions. However that was beyond the scope of the current observational study. On the other hand, among 5 such WLI-indistinct lesions with equivocal margins, 4 were classified as LPDED- $(++)$, while 1 was LPDED-(+). In this regard, it is expected that this ALA-mediated PDD method would be useful for determining the tumor extent, providing additional information about demarcation during luminal therapies, as well as helping to identify such indistinct neoplastic lesions as those shown in the Figures.

The other limitation of this study is that the histopathological types of all lesions were adenoma or differentiated adenocarcinoma. There, between the LPDEDpositive and negative groups, there was a significant difference in histopathology [14]. In our prior study, among 33 lesions, 26 tumors were detectable by LPDED with the first prototype Sie-P1. These were exceptionally differentiated adenocarcinomas and adenomas, and the fluorescent intensity was higher in differentiated adenocarcinomas than in signetring cell carcinomas. Thus, the sensitivity was $100 \%$. However, that might be attributable to the inclusion criteria of ESD; cases of adenoma or differentiated adenocarcinoma with nominal risks of lymph node metastasis [3] were enrolled in this study. As this was a pilot study, only patients with EGC and adenoma were included; therefore, we could only demonstrate the sensitivity of the LPDED with the Sie-P2 system. The diagnostic ability of the LPDED system in patients with and without disease will be investigated in a future phase II study.

Moreover, there was a substantial variation in the intensity of fluorescence among the 16 LPDED-positive tumors, despite the lack of significant differences in age, sex, macroscopic type, tumor size, tumor location, and histopathological types of differentiated adenocarcinoma or adenoma. The underlying mechanism by which 5-ALA-mediated PpIX accumulates differentially remains uncertain, but it could reflect the diversity in histological types of gastric tumors. PpIX is the final intermediate in the heme biosynthesis pathway that is formed by protoporphyrinogen IX oxidase (PPOX), and PPOX plays an essential catalyzing enzymatic role in this pathway, directly leading to enhanced PpIX biosynthesis [9,14]. We confirmed that the PPOX expression was higher in intestinal types of differentiated adenocarcinoma, as well as adenoma, than in the diffuse type of sig EGCs [14]. The overexpression could be involved in the fluorescence intensity of LPDED, possibly reflecting histopathological features of intestinal types, including adenomas. Further studies including other histopathological types of EGC are needed to confirm the utility of the second-generation prototype endoscope for LPDED.

In conclusion, the second-generation endoscopic system for LPDED was able to detect differentiated EGC and highgrade adenoma. The Sie-P2 could get brighter image pictures than the first-generation prototype, Sie-P1, but optimization of image data processing is necessary to achieve more contrasted and secure detection of EGC, and a possible ability to detect other histological types of EGC.

\section{Summary Box}

\section{What is already known:}

- The reported detection rates of early gastric cancer (EGC) are not sufficiently high

- There have been advances in image enhanced endoscopy, but such advanced imaging modalities require endoscopic skills and experience with expertise

- For objective and easy identification for nonexperts, we developed laser-based photodynamic endoscopic diagnosis (LPDED) with 5-aminolevulinic acid (5-ALA) using the first prototype endoscope system, Sie-P1; however, because of the need for a cut-filter, the captured images were dark, a potential drawback

\section{What the new findings are:}

- A novel prototype endoscope system, Sie-P2, for 5-ALA-based LPDED can improve the previous dark imaging, as it does not require a cut-filter

- The new LPDED system was useful in detecting 16 lesions that included differentiated EGC and adenoma (sensitivity 100\%) without substantial adverse events

- This observation study of LPDED modification opens the door to further research to optimize the image data processing for more contrasted and secure detection of the gastric neoplastic lesions 


\section{References}

1. Bray F, Ferlay J, Soerjomataram I, Siegel RL, Torre LA, Jemal A. Global cancer statistics 2018: GLOBOCAN estimates of incidence and mortality worldwide for 36 cancers in 185 countries. $C A$ Cancer J Clin 2018;68:394-424.

2. Yamaguchi N, Isomoto H, Fukuda E, et al. Clinical outcomes of endoscopic submucosal dissection for early gastric cancer by indication criteria. Digestion 2009;80:173-181.

3. Isomoto H, Shikuwa S, Yamaguchi N, et al. Endoscopic submucosal dissection for early gastric cancer: a large-scale feasibility study. Gut 2009;58:331-336.

4. Goto O, Fujishiro M, Kodashima S, Ono S, Omata M. Outcomes of endoscopic submucosal dissection for early gastric cancer with special reference to validation for curability criteria. Endoscopy 2009;41:118-122.

5. Yamaguchi D, Kodashima S, Fujishiro M, et al. Evaluation of imageenhanced endoscopic technology using advanced diagnostic endoscopy for the detection of early gastric cancer: a pilot study. Endosc Int Open 2017;5:E825-E833.

6. Shimodate Y, Mizuno M, Doi A, et al. Gastric superficial neoplasia: high miss rate but slow progression. Endosc Int Open 2017;5:E722-E726.

7. Menon S, Trudgill N. How commonly is upper gastrointestinal cancer missed at endoscopy? A meta-analysis. Endosc Int Open 2014;2:E46-E50.

8. Isomoto $\mathrm{H}$, Nanashima $\mathrm{A}$, Senoo $\mathrm{T}$, et al. In vivo fluorescence navigation of gastric and upper gastrointestinal tumors by 5-aminolevulinic acid mediated photodynamic diagnosis with a laser-equipped video image endoscope. Photodiagnosis Photodyn Ther 2015;12:201-208.
9. Stepp H, Stummer W. 5-ALA in the management of malignant glioma. Lasers Surg Med 2018;50:399-419.

10. Yamamichi G, Nakata W, Tani M, et al. High diagnostic efficacy of 5-aminolevulinic acid-induced fluorescent urine cytology for urothelial carcinoma. Int J Clin Oncol 2019;24:1075-1080.

11. Nakai Y, Inoue K, Tsuzuki T, et al. Oral 5-aminolevulinic acidmediated photodynamic diagnosis using fluorescence cystoscopy for non-muscle-invasive bladder cancer: A multicenter phase III study. Int J Urol 2018;25:723-729.

12. Kitada M, Ohsaki Y, Matsuda Y, Hayashi S, Ishibashi K. Photodynamic diagnosis of pleural malignant lesions with a combination of 5-aminolevulinic acid and intrinsic fluorescence observation systems. BMC Cancer 2015;15:174.

13. Xiao Q, Chen T, Chen S. Fluorescent contrast agents for tumor surgery. Exp Ther Med 2018;16:1577-1585.

14. Kurumi H, Kanda T, Kawaguchi K, et al. Protoporphyrinogen oxidase is involved in the fluorescence intensity of 5-aminolevulinic acid-mediated laser-based photodynamic endoscopic diagnosis for early gastric cancer. Photodiagnosis Photodyn Ther 2018;22:79-85.

15. Japanese Gastric Cancer Association. Japanese classification of gastric carcinoma: $3^{\text {rd }}$ English edition. Gastric Cancer 2011;14:101-112.

16. Park DI, Rhee PL, Kim JE, et al. Risk factors suggesting malignant transformation of gastric adenoma: univariate and multivariate analysis. Endoscopy 2001;33:501-506.

17. Nakamura M, Nishikawa J, Hamabe K, et al. Preliminary study of photodynamic diagnosis using 5-aminolevulinic acid in gastric and colorectal tumors. World J Gastroenterol 2015;21:6706-6712.

18. Nakamura T, Oinuma T, Yamagishi H, Masuyama H, Terano A. Evaluation of a novel high-resolution magnifying videoendoscope that is capable of photodynamic diagnosis and therapy for gastric cancer. Photodiagnosis Photodyn Ther 2015;12:115-122. 